Tailoring the diameter and density of self-catalyzed GaAs nanowires on silicon

This content has been downloaded from IOPscience. Please scroll down to see the full text. 2015 Nanotechnology 26105603

(http://iopscience.iop.org/0957-4484/26/10/105603)

View the table of contents for this issue, or go to the journal homepage for more

Download details:

This content was downloaded by: annafm

IP Address: 128.178.23.111

This content was downloaded on 18/02/2015 at 04:45

Please note that terms and conditions apply. 


\title{
Tailoring the diameter and density of self- catalyzed GaAs nanowires on silicon
}

\author{
Federico Matteini ${ }^{1}$, Vladimir G Dubrovskii ${ }^{2,3,4}$, Daniel Rüffer ${ }^{1}$, \\ Gözde Tütüncüoğlu ${ }^{1}$, Yannik Fontana ${ }^{1}$ and Anna Fontcuberta I Morral ${ }^{1}$ \\ ${ }^{1}$ Laboratoire Matériaux Semiconducteurs, École Politechnique Fédérale de Lausanne, Switzerland \\ ${ }^{2}$ St. Petersburg Academic University, Khlopina 8/3, 194021 St. Petersburg, Russia \\ ${ }^{3}$ Ioffe Physical Technical Institute of the Russian Academy of Sciences, Politekhnicheskaya 26, 194021 St. \\ Petersburg, Russia \\ ${ }^{4}$ ITMO University, Kronverkskiy pr. 49, 197101 St. Petersburg, Russia
}

E-mail: anna.fontcuberta-morral@epfl.ch

Received 9 October 2014, revised 5 December 2014

Accepted for publication 13 January 2015

Published 17 February 2015

\begin{abstract}
Nanowire diameter has a dramatic effect on the absorption cross-section in the optical domain. The maximum absorption is reached for ideal nanowire morphology within a solar cell device. As a consequence, understanding how to tailor the nanowire diameter and density is extremely important for high-efficient nanowire-based solar cells. In this work, we investigate mastering the diameter and density of self-catalyzed GaAs nanowires on $\mathrm{Si}(111)$ substrates by growth conditions using the self-assembly of Ga droplets. We introduce a new paradigm of the characteristic nucleation time controlled by group III flux and temperature that determine diameter and length distributions of GaAs nanowires. This insight into the growth mechanism is then used to grow nanowire forests with a completely tailored diameter-density distribution. We also show how the reflectivity of nanowire arrays can be minimized in this way. In general, this work opens new possibilities for the cost-effective and controlled fabrication of the ensembles of self-catalyzed III-V nanowires for different applications, in particular in next-generation photovoltaic devices.
\end{abstract}

S Online supplementary data available from stacks.iop.org/NANO/26/105603/mmedia

Keywords: GaAs nanowires, self-catalyzed growth, nucleation, growth modeling, light absorption

(Some figures may appear in colour only in the online journal)

\section{Introduction}

Semiconductor nanowires are interesting as building blocks for next-generation optoelectronic and electronic technologies [1-5]. Within a wide range of III-V compounds used to form these structures, GaAs is among the most promising materials for photovoltaic applications [6]. Furthermore, GaAs nanowire-based solar cells can be grown on $\mathrm{Si}$, easily resulting in a dual-junction device [7]. Proof-of-concept radial p-i-n GaAs nanowire solar cells have been demonstrated in the past [813]. Recently, it has been shown that the achievement of high efficiencies can only be obtained after the accurate design of the individual junction, the nanowire diameter, and density so that light absorption and conversion are maximized [14, 15]. Pre-patterning the substrate would be an approach to control density. However, such a technique is technologically demanding and presents several open challenges [15-17]. Furthermore, a regular array would not work on all incident angles due to diffraction effects [18]. We propose here a more cost-effective alternative: the self-assembly of nanowires. We will show how understanding the initial stages of growth provides the tools to control the dimensions of the nanowires on the substrate.

To the best of our knowledge, relatively few works published so far report on the distribution of nanowire density and diameters [19-23]. An original approach for controlling 
the nanowire density makes use of droplet epitaxy techniques [19]. This method is very efficient for controlling the density and diameter of $\mathrm{Ga}$ droplets that initiate $\mathrm{GaAs}$ nanowires. However, when it comes to the transition from droplets to nanowires, the yield is not yet $100 \%$. Additionally, uncontrolled secondary nucleation changes the predetermined nanowire density and diameter. Some groups have reported on the substrate temperature effect on the nanowire diameter and density [22, 24]. However, no comprehensive model was proposed to grasp the fundamental mechanism and thereby tailor the nanowire diameter and density in an effective manner.

Consequently, in this work we study the impact of the growth conditions on the morphology and density of selfassembled GaAs nanowires on $\mathrm{Si}$ (111). An understanding of the underlying mechanism is used to achieve diameter-density combinations 'à la carte', allowing one to tailor light absorption of the self-assembled nanowire arrays. We start by showing the general influence of the substrate temperature and Ga flux over the nanowire density, diameter and length distributions. This is followed by presenting a model that reveals the underlying mechanisms. The model is then used to develop an alternative growth approach based on two steps of Ga fluxes to tailor at will the nanowire diameter and density. Finally, we show how tailoring the nanowire diameter-density can be used to increase the overall solar light absorption.

\section{Experimental details}

Our samples were grown by molecular beam epitaxy (MBE) on $\mathrm{Si}(111)$ substrates covered with a native oxide layer. The surface roughness of the substrates was around $0.4 \pm 0.2 \mathrm{~nm}$ as measured by atomic force microscopy. Growths were carried out with simultaneous deposition of $\mathrm{Ga}$ and $\mathrm{As}_{4}$. The range of Ga fluxes extended from $0.03 \mathrm{~nm} \mathrm{~s}^{-1}$ to $0.11 \mathrm{~nm} \mathrm{~s}^{-1}$, as calibrated by reflection high-energy electron diffraction (RHEED). The As flux was kept constant in all the experiments at $2.5 \times 10^{-6}$ Torr, and was calibrated by a beam flux monitor gauge. The range of temperatures used went from $\sim 600^{\circ} \mathrm{C}$ to $645^{\circ} \mathrm{C}$, calibrated by a pyrometer that measures the emissivity of the $\mathrm{Si}$ substrate.

\section{Results}

\subsection{Impact of growth conditions}

In the first set of experiments, the substrate temperature was varied from $604{ }^{\circ} \mathrm{C}$ up to $T=643{ }^{\circ} \mathrm{C}$, whereas the Ga growth rate was kept constant at $V=0.11 \mathrm{~nm} \mathrm{~s}^{-1}$.

Figures 1(a)-(b) show the distribution of nanowire length and diameter for three different temperatures. The statistics are the result of measuring a total of at least 100 nanowires. The lines show theoretical fits by the model presented hereafter. The increase of temperature decreases the nanowire length from $\sim 4.5 \mu \mathrm{m}$ to $\sim 2.8 \mu \mathrm{m}$ (see figure 1(a)), and
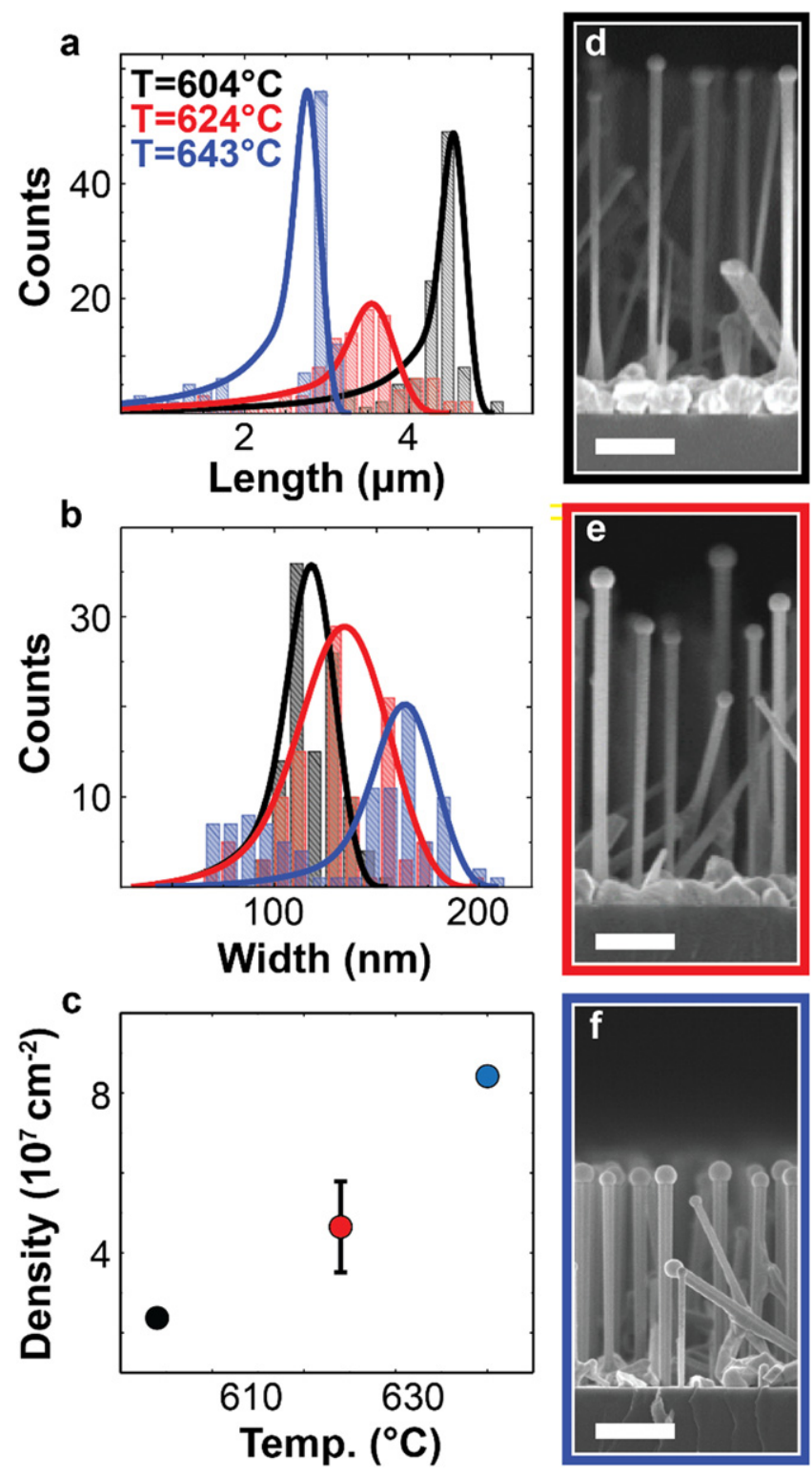

Figure 1. Length (a) and width $(d=2 R)$ (b) distributions of selfcatalyzed GaAs nanowires grown at a fixed Ga flux of $0.11 \mathrm{~nm} \mathrm{~s}^{-1}$ and three different temperatures $\left(\mathrm{T}=604{ }^{\circ} \mathrm{C}, \mathrm{T}=624^{\circ} \mathrm{C}\right.$ and $\mathrm{T}=643^{\circ} \mathrm{C}$ ) for $60 \mathrm{~min}$. The lines in (a) and (b) are the fits obtained from the model presented in the text (for further details, see the supplementary information), with the parameters summarized in table 1. In (c) the evolution of nanowire density over different substrate temperatures is reported. The scanning electron micrographs of the nanowires grown at the three different temperatures are, respectively, shown in (d), (e) and (f). The scalebar is 1 micrometer.

increases the diameters from $\sim 100 \mathrm{~nm}$ up to $\sim 160 \mathrm{~nm}$, (see figure $1(\mathrm{~b}))$.

Figure 1(c) shows the density dependence over temperature (from $2.4 \times 10^{7}$ nanowires $/ \mathrm{cm}^{2}$ to $8.4 \times 10^{7}$ nanowires $/ \mathrm{cm}^{2}$ ), and figures 1(d)-(f) show typical scanning electron micrographs of the samples. In general, higher substrate temperatures result in a reduction of the length and an increase in the diameter. Higher substrate temperatures also result in a reduction of polycrystalline GaAs islands, also 

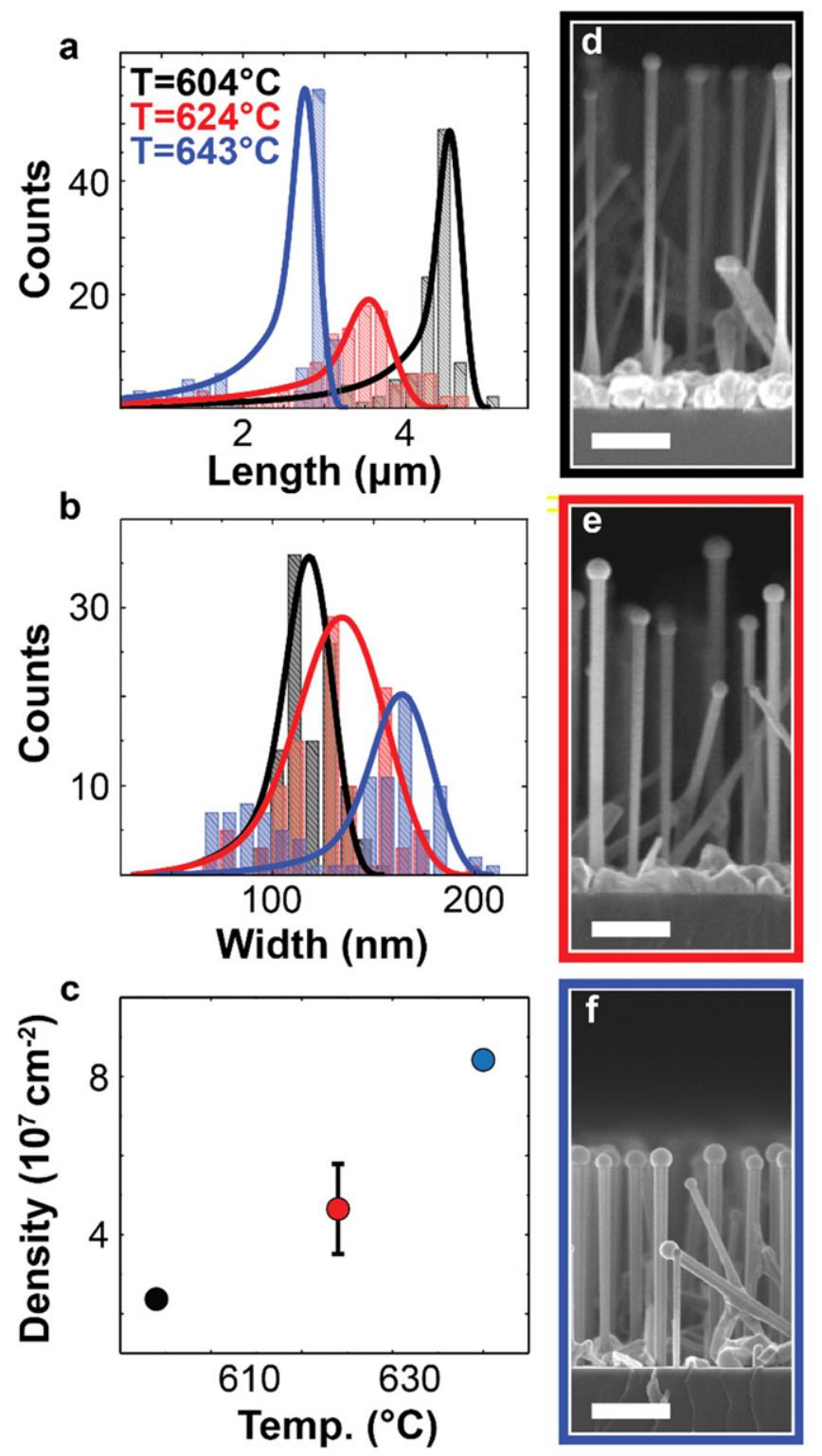

Figure 2. Length (a) and width (b) distributions of self-catalyzed $\mathrm{GaAs}$ nanowires grown at a fixed substrate temperature of $643{ }^{\circ} \mathrm{C}$ and three different $\mathrm{Ga}$ fluxes $\left(\mathrm{V}_{1 \mathrm{~s}}=0.11 \mathrm{~nm} \mathrm{~s}^{-1}, \mathrm{~V}_{1 \mathrm{~s}}=0.075 \mathrm{~nm} \mathrm{~s}^{-1}\right.$ and $\mathrm{V}_{1 \mathrm{~s}}=0.05 \mathrm{~nm} \mathrm{~s}^{-1}$ ) for $60 \mathrm{~min}$. In (c) the evolution of nanowire density over different $\mathrm{Ga}$ fluxes is reported. The lines in (a) and (b) are the fits obtained from the model, with the parameters summarized in table 1 . (d), (e) and (f) show the respective scanning electron micrographs of the nanowires grown at the three different Ga fluxes. The scalebar is 1 micrometer.

called parasitic growth, while the nanowire density increases. In the second experiment, we looked at the nanowire diameter and length distribution under varying $\mathrm{Ga}$ flux. We chose the substrate temperature of $643{ }^{\circ} \mathrm{C}$, which was found to minimize the parasitic growth. The Ga flux was varied from $0.11 \mathrm{~nm} \mathrm{~s}^{-1}$ to $0.05 \mathrm{~nm} \mathrm{~s}^{-1}$. The results are shown in figure 2 , which displays the corresponding length and diameter distributions. The decrease of $\mathrm{Ga}$ flux produces a drop in nanowire length, broadening its distribution (see figure 2(a)), although without presenting a clear trend. The diameter distribution is very sensitive to the Ga flux: the mean diameter shrinks from $\sim 160 \mathrm{~nm}$ to $\sim 50 \mathrm{~nm}$ as the Ga flux is decreased (see figure 2(b)). Figure 2(c) shows the nanowire density at different $\mathrm{Ga}$ fluxes. The decrease of $\mathrm{Ga}$ flux results in a broadening of the length distributions and reduction of the mean diameter, as well as a fall in density. Figures 2(d)-(f) show the typical scanning electron micrographs of as-grown GaAs nanowire samples. A pronounced decrease in nanowire diameter and density at decreasing Ga fluxes is observed. While the increase of the nanowire diameter with the Ga flux is expected and was reported earlier in [9], narrowing of the diameter distribution has not been discussed before.

\subsection{The model}

A common feature of the nanowire morphologies presented here is rather broad distributions of length and diameter. The nanowire density seems to be directly linked to the growth temperature and Ga flux. In contrast to the standard vaporliquid-solid (VLS) growth procedures where metal catalyst particles are prepared before the material deposition [23, 2528], our self-assembled approach makes use of Ga droplets that form concomitantly with nanowires [29]. Therefore, rather than having all nanowires emerging instantly at $t=0$, different nanowires nucleate and start growing vertically at different moments of time $0 \leqslant t_{0} \leqslant t$, where $t$ is the total deposition time. This important feature requires a careful investigation of time-dependent nucleation of nanowires and nanowire statistics, a problem that, to the best of our knowledge, has not been addressed so far. On the other hand, self-catalyzed III-V nanowires usually grow not only vertically, but also extend radially [30, 31]. This is due to an excessive group III influx into the reservoir in the Ga droplet. Without accounting for different starting times for growth as well as the radial extension, the deterministic length-radius, length-time and radius-time correlations (which usually assume instantaneous nucleation from preexisting catalyst particles and a time-independent nanowire radius, defined by the size of these particles [23, 26, 27]) would not give the adequate description of self-catalyzed growth in our experimental conditions.

Consequently, in our modeling we consider the random nucleation of $\mathrm{Ga}$ droplets (which then act as nanowire growth seeds with a certain yield coefficient [32]) occurring alongside the nanowire growth itself. The model is illustrated in figure 3(a) and described in the following. The gallium and arsenic species arrive onto the substrate surface, and the Ga droplet surfaces at the rates $I_{3}$ and $I_{5}$, respectively, measured in atoms per unit time per unit area. When Ga and As adatoms meet on the surface, they form the parasitic (polycrystalline) GaAs islands at the rate $K_{35}\left(\mathrm{~nm}^{2} \mathrm{~s}^{-1}\right)$. Dimerization of two Ga adatoms at the rate $K_{3}$ will subsequently lead (with a certain probability) to the nucleation of $\mathrm{Ga}$ nanodroplets and ultimately nanowires. Both processes are expected to be associated with the formation of 'craters' or even the openings penetrating through the oxide toward the substrate surface [29, 33, 34]. Therefore, the rate constants $K_{3}$ and $K_{35}$ should be orders of magnitude lower than that given by the effective 

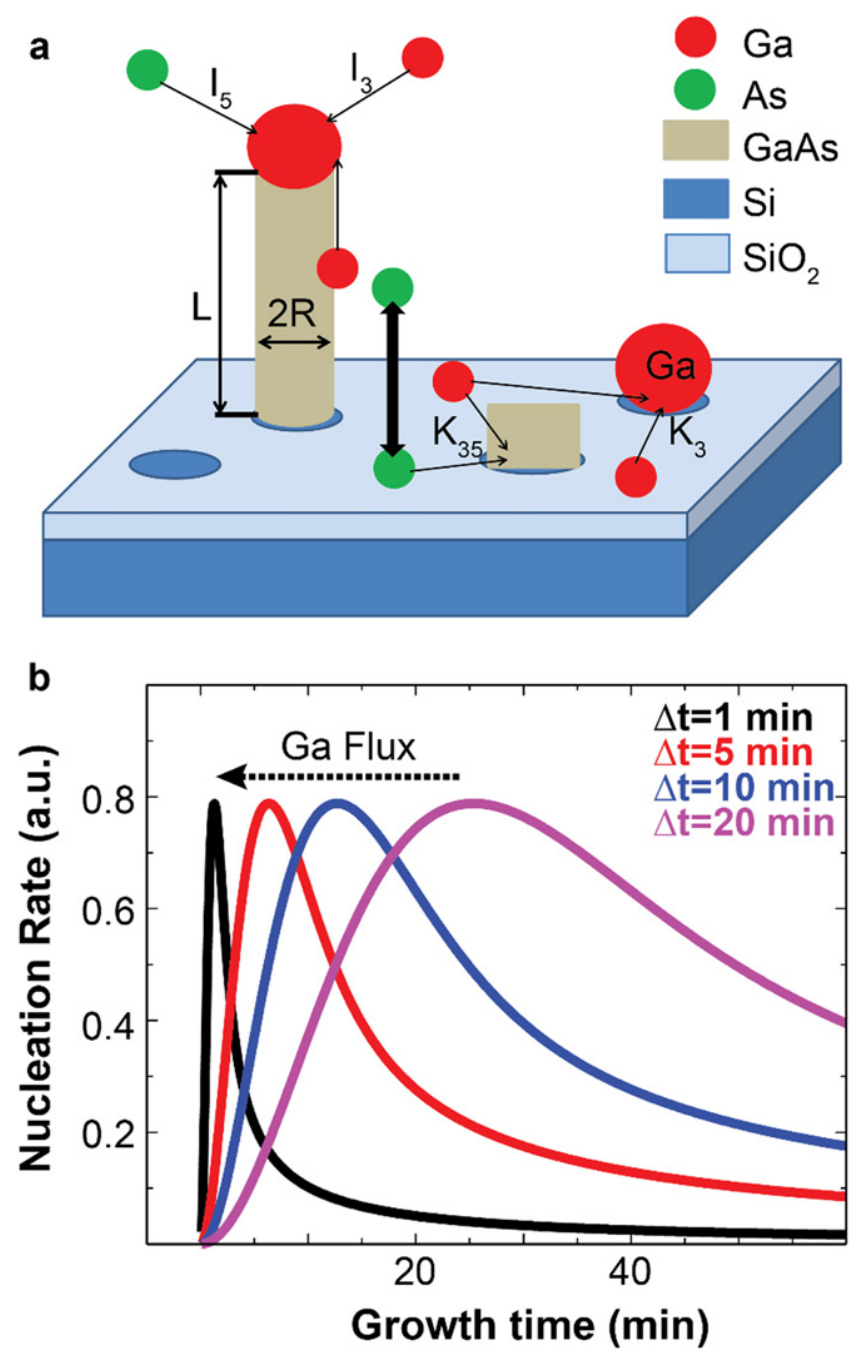

Figure 3. (a) Schematics of different kinetic processes considered within the growth model: (i) formation of Ga droplets from two $\mathrm{Ga}$ adatoms in the 'craters'; (ii) formation of parasitic GaAs islands from $\mathrm{Ga}$ and As atoms in the craters and (iii) nanowire growth catalyzed by Ga droplets, with the model parameters described in the text. In (b) the time dependence of the nucleation rate of Ga droplets at different nucleation times $\Delta t$ is shown.

Ga diffusivities $\left(\sigma_{3} D_{3}\right.$ and $\sigma_{35} D_{3}$, with $\sigma$ as the corresponding capture coefficients [35-37]).

Within the frame of the irreversible growth model, that is, with neglect of decay of both $\mathrm{Ga}-\mathrm{Ga}$ and Ga-As surface dimers [35-37], the set of kinetic equations for the surface density of Ga droplets $\left(N_{3}\right)$, parasitic GaAs islands $\left(N_{35}\right)$ and $\mathrm{Ga}$ adatoms $\left(n_{3}\right)$ can be written as

$$
\begin{aligned}
& \frac{d N_{3}}{d t}=K_{3} n_{3}^{2} ; \\
& \frac{d N_{35}}{d t}=K_{35} n_{3} n_{5} \cong K_{35} n_{3} I_{5} \tau_{5} ; \\
& \frac{d n_{3}}{d t}=I_{3}-K_{3} n_{3} N_{3}-K_{35} n_{3} N_{35} .
\end{aligned}
$$

Here, we assume that the kinetic growth constants are the same for differently sized surface clusters, as in [36] and [37]. The first equation shows that the number of $\mathrm{Ga}$ droplets increases due to the Ga-Ga dimerization, with $K_{3} n_{3}^{2}$ as the nucleation rate in irreversible growth [37]. The second equation gives the nucleation rate of parasitic GaAs islands, where we assume that the unknown concentration of As adatoms quickly equilibrates with the vapor atomic flux $I_{5}$ : $n_{5} \cong I_{5} \tau_{5}$, with $\tau_{5}$ being the characteristic lifetime of As. This $\tau_{5}$ is expected to decrease very steeply with increasing surface temperature due to enhanced desorption of As. The third equation gives the time dependence of the Ga adatom concentration, which increases due to the vapor influx and decreases when the Ga adatoms attach to either the Ga droplets or GaAs islands.

In the large time limit where $d n_{3} / d t \rightarrow 0$, the Ga adatom concentration is given by the dynamic balance between the vapor flux and the adatom consumption by the growing islands and droplets:

$$
n_{3}=\frac{I_{3}}{K_{3} N_{3}+K_{35} N_{35}} \cong \frac{I_{3}}{K_{35} N_{35}} .
$$

In all our samples, the surface area covered by parasitic GaAs is much larger than that covered by the nanowires (and hence the $\mathrm{Ga}$ droplets). Thus, the limiting case of $K_{3} N_{3} \ll K_{35} N_{35}$ should apply uniformly in these conditions. Using this $n_{3}$ in equation (1) for $N_{3}$ and $N_{35}$, and integrating over, we obtain the large time asymptotes of the island and droplet densities:

$$
N_{35}=\left(2 I_{3} I_{5} \tau_{5} t\right)^{1 / 2} ; \quad N_{3}=\frac{K_{3} I_{3}}{2 K_{35}^{2} I_{5} \tau_{5}} \ln \left(t / t_{0}\right) .
$$

We can see that the concentration of parasitic GaAs islands scales with time as $\mathrm{t}^{1 / 2}$, whereas the Ga droplet concentration increases with time only logarithmically (i.e., almost saturates at large enough $t$ ). As mentioned earlier, each $\mathrm{Ga}$ droplet gives rise to a newly formed nanowire with the probability $\chi$. The nanowire surface density is thus given by

$$
N_{n w}=\chi N_{3},
$$

where the yield $\chi$ may depend on temperature and the Ga flux.

Despite its simplicity, our nucleation model is capable of explaining the major experimental trends:

(i). Since the leading temperature dependence of the prefactors in equation (3) should be determined by a rapidly decreasing As lifetime $\tau_{5}$, parasitic growth decreases and nanowire density increases when the surface temperature is raised, as experimentally observed in figure 1.

(ii). Both $N_{35}$ and $N_{\text {nw }}$ increase at higher $\mathrm{Ga}$ flux, as experimentally observed in figure 2 . Since the theoretical dependence of the Ga droplet density on $I_{3}$ is linear and the observed experimental correlation $N_{n w}\left(I_{3}\right)$ is superlinear, it can be concluded that the yield coefficient increases with Ga flux.

By further developing the model based on some earlier results for the growth kinetics of GaAs nanowires [31, 38, 39] and the nucleation equations [26, 40] (all the details are provided in the supplementary information), we get the 
Table 1. Theoretical parameters used for fitting the length and width distributions for different samples.

\begin{tabular}{llcccccccc}
\hline$T\left({ }^{\circ} \mathrm{C}\right)$ & $V\left(\mathrm{~nm} \mathrm{~s}^{-1}\right)$ & $L_{\max }(\mathrm{nm})$ & $\Delta L(\mathrm{~nm})$ & $\Delta t(\min )$ & $2 R_{\max }(\mathrm{nm})$ & $2 \Delta R(\mathrm{~nm})$ & $C\left(\mathrm{~nm} \mathrm{~min}^{-1}\right)$ & $\left.A(\mathrm{~nm} \mathrm{~min})^{-1}\right)$ & $2 R_{c}(\mathrm{~nm})$ \\
\hline 604 & 0.11 & 5050 & 380 & 4.5 & 145 & 35 & 84 & -2.76 & 200 \\
624 & 0.11 & 4500 & 710 & 9.5 & 195 & 65 & 75 & -2.41 & 205 \\
643 & 0.11 & 3400 & 450 & 7.9 & 210 & 48 & 57 & -1.45 & - \\
643 & 0.075 & 3200 & 1550 & 29 & 129 & 42 & 53 & -1.55 & 210 \\
643 & 0.05 & 3700 & 1550 & 25 & 105 & 38 & 62 & -1.73 & 130 \\
643 & $0.11 / 0.075$ & 2500 & 650 & 15.5 & 145 & 42 & 42 & $-1.45 /-1.69$ & 190 \\
643 & $0.11 / 0.05$ & 2500 & 650 & 15.5 & 140 & 50 & 42 & $-1.45 /-1.86$ & 190 \\
643 & $0.11 / 0.027$ & 2500 & 650 & 15.5 & 135 & 60 & 42 & $-1.45 /-2.03$ & 190 \\
\hline
\end{tabular}

corresponding nanowire nucleation rate in the form

$$
J_{n w}=\frac{d N_{n w}}{d t}=\chi J_{0} g^{2}\left[(t / \Delta t)^{3 / 2}\right] .
$$

Here, $J_{0}$ is the known normalization constant and $\Delta t=4^{4 / 3} /[3$ $\left.\left(K_{3} I_{3}\right)^{1 / 2}\right]$ is the characteristic nucleation time interval for $\mathrm{Ga}$ droplets. The universal function $g(y)$ is given by

$$
g(y)=e^{-y} \int_{0}^{y} d x \frac{e^{x}}{x^{1 / 3}}
$$

Hence, the nanowire nucleation rate varies at different characteristic nucleation times, as shown in figure 3(b): the larger the $\Delta t$, the more spread in time and the more delayed is the nucleation rate. The variations in the characteristic nucleation time is controlled by $I_{3}$, the group III flux. In other words, the initial $\mathrm{Ga}$ flux $I_{3}$ determines the distribution of diameter and lengths: a larger Ga flux yields a narrower diameter and length distributions (i.e., more uniform), since the nucleation rate over time is narrower for smaller $\Delta t$ (see figure 3(b)). Instead, a lower Ga flux produces an upturn in $\Delta t$ that broadens the nucleation pulse over time, resulting in wider diameter and length distributions. The model presented earlier explains very well the results of the Ga flux set of experiments, where broader distributions are observed at diminishing Ga flux.

In order to understand the length and radius distributions of self-catalyzed nanowires and to describe their evolution with the growth time, we now consider the nanowire growth itself. According to the model described in detail in [31], the nanowire elongation rate is proportional to the difference between the impinging atomic As flux and desorption flux, while the surface diffusion of As can be neglected [38, 39]. In contrast, $\mathrm{Ga}$ is not expected to desorb from the droplet at temperatures below $650{ }^{\circ} \mathrm{C}$, but can easily migrate from the nanowire sidewalls to the droplet. The increase of the droplet volume is determined by the effective Ga-to-As imbalance. In fact, the decrease of Ga flux leads to smaller diameters, as already reported in figure 2(b), whereas a temperature increase produces a diameter expansion (see figure 1(b)), related to the decrease in As lifetime $\tau_{5}$.

These considerations yield our main results for the length $(L)$ and radius $(R)$ distributions of self-induced nanowires given by

$$
f\left(L, L_{\max }\right)=\text { const } \times g^{2}\left[\left(\frac{L_{\max }-L}{\Delta L}\right)^{3 / 2}\right]
$$

$$
F\left(R, R_{\max }\right)=\text { const } \times \frac{1}{B / R-|A|} g^{2}\left[\left(\frac{R_{\max }-R}{\Delta R}\right)^{3 / 2}\right] .
$$

Here, $g$ is the same function as in equation (6), whereas $L_{\max }$ and $R_{\max }$ are the maximum length and the maximum radius of the nanowires having nucleated at the very beginning of GaAs deposition. The parameters $\Delta L$ and $\Delta R$ describe the widths of the length and radius distributions. Both $\Delta L$ and $\Delta R$ are proportional to $\Delta t$. The $A$ and $B$ in equation (8) are the kinetic coefficients that can be obtained by fitting the time dependences of the mean length and diameter (see supplementary information). As usual in the deterministic growth theories [26, 37, 40], the size distributions reflect the shape of the time-dependent nucleation rate, inverted in such a way that zero moment of time corresponds to the maximum size of nanowires. Full details of derivation of the key in equations (7) and (8) are given in the supplementary information. Clearly, with the measured $L_{\max }, R_{\max }, A$ and $B$, theoretical length and diameter distributions of nanowires contain only one fitting parameter: the corresponding distribution width $(\Delta L$ or $\Delta R$ ), which is determined by the random character of nucleation. The parameters used for fitting the experimental length and diameter histograms in figures 1 , 2 and 6 are listed in table 1 , in which the critical radius $R_{\mathrm{c}}$ is defined as $R_{\mathrm{c}}=B /|A|, V=\Omega_{35} I_{3} \cos \alpha_{3}$ is the Ga deposition rate, $\Omega_{35}=0.0452 \mathrm{~nm}^{3}$ is the elementary volume per GaAs pair in the solid state and $\alpha_{3}=45^{\circ}$ is the incidence angle of the Ga beam with respect to the substrate. The parameter $C$ is obtained from $L_{\max }=C t$ for the total growth time $t=60 \mathrm{~min}$ and the characteristic nucleation time $\Delta t$ from $\Delta L=\mathrm{C} \Delta t$ (see the supplementary information for the details).

Figure 4 summarizes the effect of Ga flux variations at a constant temperature and the effect of temperature variations at a constant $\mathrm{Ga}$ flux over the nanowire diameter and density. By augmenting $\mathrm{Ga}$ flux, diameter and density increase (figure 4, blue arrow); a similar behavior is observed in the case of temperature increase (figure 4, black arrow). Therefore, within the classic growth approach where the Ga flux is kept constant during growth, small nanowires $(<100 \mathrm{~nm})$ can 


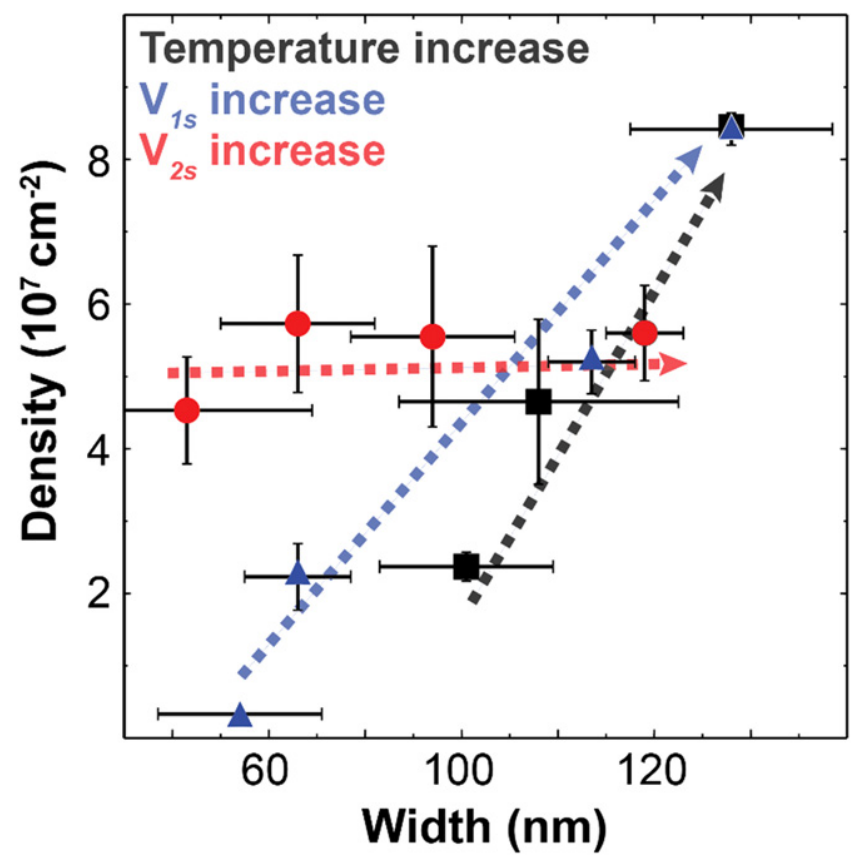

Figure 4. Diameter-density correlation of (i) one-step Ga series (blue triangles), (ii) temperature series (black squares) and (iii) two-step Ga series (red circles). (i) The increase in Ga flux (blue arrow) results in larger diameters and denser nanowire forests. In a similar fashion, (ii) higher growth temperature increases diameter and density (black arrow). (iii) In the case of two-step Ga flux series, the decrease of the Ga flux at the second step (red arrow) lowers down the nanowire diameters without changing the density values.

be achieved only at the compromise of low density, and vice versa (see figure 4).

\subsection{Alternative two-step approach}

We now turn to the implementation of our model for the reverse engineering of diameter, length and density. In order to combine a high density of nanowires with small diameters, we grew nanowires in a two-step fashion. We initiated the growth at a high Ga rate $\left(0.11 \mathrm{~nm} \mathrm{~s}^{-1}\right)$ in view of achieving a high nucleation rate (i.e., high nanowire density). Then after 30 min of growth, we decreased the Ga rate in order to suppress supplementary nanowire initiation and to decrease the nanowire diameter by consuming some additional $\mathrm{Ga}$ from the droplet.

Figures 5(a) and (b) show the corresponding length and diameter distribution, respectively. The nanowire length does not seem to be affected by decreasing the Ga flux at the second growth step. Conversely, the diameter progressively diminishes as the Ga flux at the second step is decreased (from $\sim 90 \mathrm{~nm}$ to $\sim 40 \mathrm{~nm}$ ). Figure $5(\mathrm{c}$ ) displays the nanowire densities at different Ga fluxes at the second step, showing that density is not affected by the Ga flux variations. Figures 5(d)-(f) show the typical scanning electron micrographs of the as-grown samples, where the diameter decreases at diminishing Ga flux at the second step is clearly observed.

The diameter-density results for the two-step Ga fluxes are also shown in figure 4 (red arrow) in comparison with the single-step Ga flux series and with the temperature series.
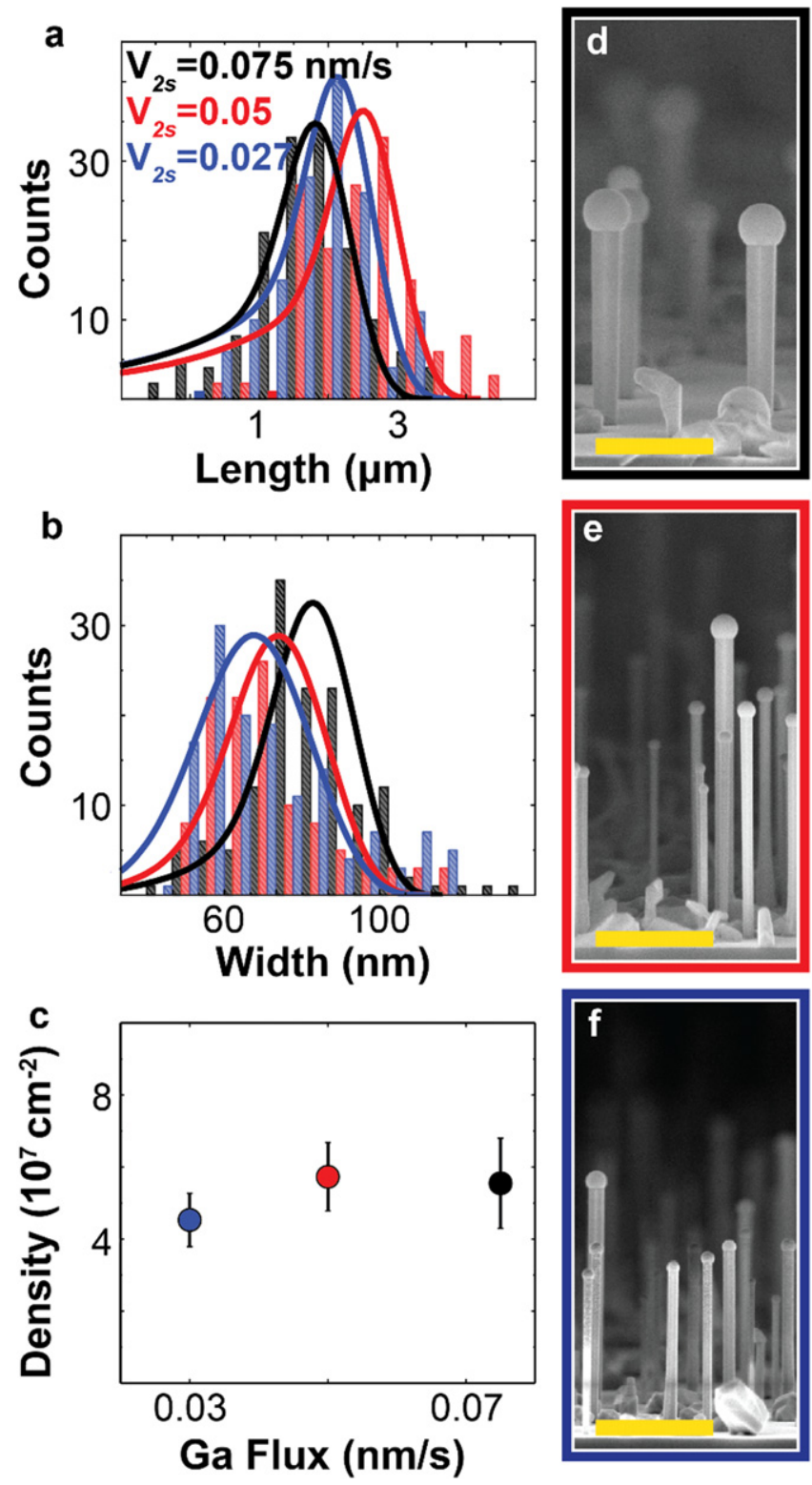

Figure 5. Length (a) and diameter (b) distributions of self-catalyzed GaAs nanowires grown at a fixed temperature of $643{ }^{\circ} \mathrm{C}$ and initial Ga flux $\left(\mathrm{V}_{1 \mathrm{~s}}=0.11 \mathrm{~nm} \mathrm{~s}^{-1}\right)$ for the first $30 \mathrm{~min}$, but different $\mathrm{Ga}$ fluxes $\left(\mathrm{V}_{2 \mathrm{~s}}=0.075 \mathrm{~nm} \mathrm{~s}^{-1}, \mathrm{~V}_{2 \mathrm{~s}}=0.05 \mathrm{~nm} \mathrm{~s}^{-1}\right.$ and $\mathrm{V}_{2 \mathrm{~s}}=0.027 \mathrm{~nm} \mathrm{~s}^{-1}$ ) for the second $30 \mathrm{~min}$. The lines in (a) and (b) are the fits obtained from the model, with the parameters summarized in table 1. In (c) the evolution of nanowire density over different Ga fluxes is reported. (d), (e) and (f) show the scanning electron micrographs of the nanowires grown, respectively, at the three different Ga fluxes for the second $30 \mathrm{~min}$. The scalebar is 1 micrometer.

Very importantly, the decrease of the Ga flux at the second step yields much smaller nanowire diameters, while the nanowire density remains relatively constant. Such a result could not be achieved by simply varying the Ga flux or temperature in the one-step procedure. The two-step Ga approach is thus very useful for tailoring the nanowire diameter and obtaining the predefined diameter-density combinations: the diameter is controlled by the Ga flux at the 


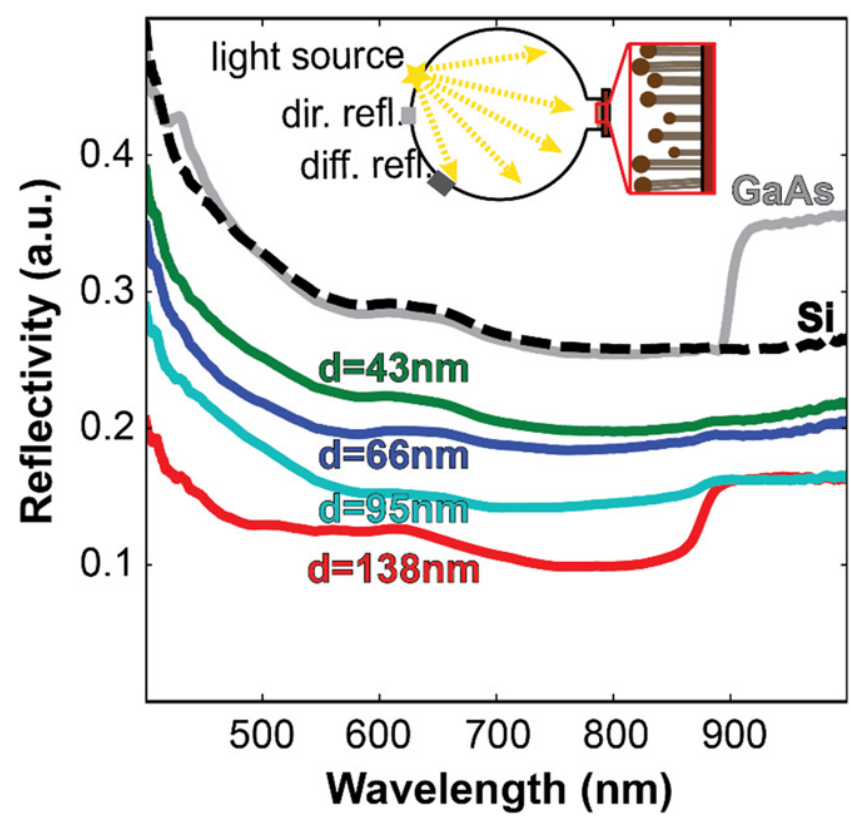

Figure 6. Reflectivity of the GaAs nanowire ensembles grown on $\mathrm{Si}$ (111) with different Ga fluxes at the second step (relating to different mean diameters $d$ ), compared to the reflectivity of $\mathrm{Si}$ and GaAs wafers. The nanowires grown at low Ga flux $\left(V=0.03 \mathrm{~nm} \mathrm{~s}^{-1}\right.$, $d=43 \mathrm{~nm}$ ) present reflectivity around $25 \%$. The increase of Ga flux at the second step progressively enhances the light absorption, which is greater than $85 \%$ for the highest Ga flux due to the increased nanowire diameter $(d=138 \mathrm{~nm})$. Around $900 \mathrm{~nm}$, the absorption decrease is observed, related to the GaAs band gap and is more pronounced for GaAs wafer and the widest nanowires with $d=138 \mathrm{~nm}$. The inset illustrates schematics of the setup used for the reflectance measurements.

second step, while the density is determined by the growth parameters during nucleation.

\subsection{Absorption measurements}

The control of nanowire diameter and density is important for the application of nanowires in solar cells. In particular, it has been shown previously that the absorption rate is only enhanced for certain diameters [13-15]. In order to extrapolate the potential of these nanowire ensembles for solar cell applications, we assess their reflectivity properties in the experiments with an integrating sphere. Figure 6 shows the results of the spectral reflectivity measurements of the GaAs nanowire ensembles with similar density and different diameter, obtained by changing the Ga rate at the second step as described earlier. The reflectivity of the nanowire samples is shown in comparison with the measured reflectivity of Si and GaAs wafers. The nanowires obtained at the Ga rate of 0.027 at the second step (with smaller diameters, $d=43 \mathrm{~nm}$ ) have a reflectivity $\sim 0.25$ in the entire spectral range. These relatively high values are expected because of the low density of nanowires and the diameter outside the absorption resonance range $[14,15]$. Interestingly, the reflectivity is reduced below 0.15 for the nanowire ensemble with the largest mean diameter $(d=138 \mathrm{~nm})$, the one obtained with a $0.085 \mathrm{~nm} \mathrm{~s}^{-1} \mathrm{Ga}$ flux at the second stage. This enhancement is obtained by just increasing the diameter of the nanowires for the same surface density.

\section{Conclusion}

We have shown that the nanowire length and diameter distributions are the consequence of a time-dependent nucleation process, which is controlled by group III flux and temperature. Within this kinetic approach involving random nucleation of $\mathrm{Ga}$ droplets and consequently GaAs nanowires, the nanowire density is established at the initial nucleation step, while the diameter can be tuned by the $\mathrm{Ga}$ flux at a later growth stage. As a result, we have demonstrated an independent control of the nanowire density and diameter over time. This method allows one to circumvent the challenge of cost-ineffective lithography for organizing the $\mathrm{Ga}$ growth seeds and to control the nanowire diameter just by the Ga flux. We have shown how the control of nanowire diameter and density determines the reflectivity, with the best values below 0.15 . This result may constitute the first step towards obtaining a nanowire-based solar cell using the fully selfassembled process. The self-assembled approach is very attractive for applications where the broad length and diameter distributions do not degenerate the required properties. More generally, our procedure combines features of the deterministic VLS growth of nanowires and random selfassembly of surface islands. We have shown that the usual assumption, an instantaneous nucleation of nanowires [17, 25, 31-33] does not work for Ga-catalyzed growth of GaAs nanowires, and could significantly change the deterministic growth picture of VLS nanowires in general.

\section{Acknowledgments}

The authors acknowledge Esther Alarcon Llado for the useful discussions and EU funding through ITN Nanoembrace Eranet contract 'Incosin' and ERC Starting Grant UpCon, and SNF funding through grant nr. 143908. VGD gratefully acknowledges financial support of the Russian Science Foundation under the grant No. 14-22-00018.

\section{References}

[1] Day A W, Thelander C, Lind E, Dick K A, Borg B M, Borgstrom M, Nilsson P and Wernersson L E 2012 Highperformance InAs nanowire MOSFETs IEEE Electron Device Lett. 33791

[2] McAlpine M C, Friedman R S, Jin S, Lin K-H, Wang W U and Lieber C M 2003 High-performance nanowire electronics and photonics on glass and plastic substrates Nano Lett. 31531

[3] Guichard A R, Barsic D N, Sharma S, Kamins T I and Brongersma M L 2006 Tunable light emission from quantum-confined excitons in TiSi2-catalyzed silicon nanowires Nano Lett. 62140 
[4] Tian B, Zheng X, Kempa T J, Fang Y, Yu N, Yu G, Huang $J$ and Liber C M 2008 Coaxial silicon nanowires as solar cells and nanoelectronic power sources Nature 449885

[5] Qian F, Li Y, Gradečak S, Park H-G, Dong Y, Ding Y, Wang Z L and Lieber C M 2008 Multi-quantum-well nanowire heterostructures for wavelength-controlled lasers Nat. Mater. 7701

[6] Shockley W and Queisser H J 1961 Detailed balance limit of efficiency of pn junction solar cells J. Appl. Phys. 32510

[7] Kandala A, Betti T and Fontcuberta I Morral A 2009 General theoretical considerations on nanowire solar cell designs Phys. Status Solidi A 1173

[8] Krogstrup P, Jørgensen H I, Heiss M, Demichel O, Hollm J V, Aagesen M, Nygard J and Fontcuberta I Morral A 2013 Single-nanowire solar cells beyond the Shockley-Queisser limit Nat. Photonics 7 306-10

[9] Colombo C, Heiss M, Grätzel M and Fontcuberta I Morral A 2009 Gallium arsenide p-i-n radial structures for photovoltaic applications Appl. Phys. Lett. 94173108

[10] Holm V J, Jørgensen I H, Krogstrup P, Nygard J, Liu H and Aagesen M 2013 Surface-passivated GaAsP singlenanowire solar cells exceeding $10 \%$ efficiency grown on silicon Nat. Commun. 41498

[11] Wu J et al 2014 Wafer-scale fabrication of self-catalyzed $1.7 \mathrm{eV}$ GaAsP core-shell nanowire photocathode on silicon substrates Nano Lett. 14 2013-8

[12] Czaban J A, Thompson D A and LaPierre R R 2009 GaAs core-shell nanowires for photovoltaic applications Nano Lett. 9148

[13] Wallentin J et al 2013 InP nanowire array solar cells achieving $13.8 \%$ efficiency by exceeding the ray optics limit Science 3391057

[14] Krogstrup P, Jørgensen H I, Heiss M, Demichel O, Holm J V, Aagesen M, Nygard J and Fontcuberta I Morral A 2013 Single-nanowire solar cells beyond the Shockley-Queisser limit Nat. Photonics 7 306-10

[15] Heiss M, Russo-Averchi E, Dalmau-Mallorquí A, Tutütüncüoğlu G, Matteini F, Rüffer D, Conesa-Boj S, Demichel O, Alarcon-Lladó E and Fontcuberta I Morral A 2014 III-V nanowire arrays: growth and light interaction Nanotechonology 25014015

[16] Munshi A M et al 2014 Position-controlled uniform GaAs nanowires on silicon using nanoimprint lithography Nano Lett. 14960

[17] Gibson S J, Boulanger J P and LaPierre R R 2013 Opportunities and pitfalls in patterned self-catalyzed GaAs nanowire growth on silicon Semicond. Sci. Technol. 28 105025

[18] Kelzenberg M D, Boettcher S W, Petykiewicz J A, Turner-Evans D B, Putnam M C, Warren E L, Spurgeon J M, Briggs R M, Lewis N S and Atwater H A 2010 Enhanced absorption and carrier collection in Si Nat. Mater. 9239

[19] Somaschini C, Bietti S, Trampert A, Jahn U, Hauswald C, Riechert H, Sanguinetti S and Geelhaar L 2013 Control over the number density and diameter of GaAs nanowires on $\mathrm{Si}$ (111) mediated by droplet epitaxy Nano Lett. 136307

[20] Bietti S, Somaschini C, Frigeri C, Fedorov A, Esposito L, Geelhaar L and Sanguinetti S 2014 Self-assisted GaAs nanowires with selectable number density on silicon without an oxide layer J. Phys. D: Appl. Phys. 47394002
[21] Giang L T T, Bougerol C, Mariette H and Songmuang R 2013 Intrinsic limits governing MBE growth of Ga-assisted GaAs nanowires on $\mathrm{Si}(111)$ J. Cryst. Growth 364118

[22] Samsonenko Y B, Cirlin G E, Khrebtov A I, Bouravleuv A D, Polyakov N K, Ulin V P, Dubrovskii V G and Werner P 2011 Semiconductors 45431

[23] Dubrovskii V G, Xu T, Lambert Y, Nys J-P, Grandidier B, Stievenard D, Chen W and Pareige P 2012 Narrowing the length distribution of Ge nanowires Phys. Rev. Lett. 108 105501

[24] Chizu R, Yamaguchi M and Amano H 2013 Growth of GaAs nanowires on Si substrate by molecular beam epitaxy under alternating supply Phys. Status Solidi C 101365

[25] Wagner R S and Ellis W C 1064 Vapor-liquid-solid mechanism of single crystal growth Appl. Phys. Lett. 489

[26] Dubrovskii V G 2014 Nucleation Theory and Growth of Nanostructures (London: Springer)

[27] Dubrovskii V G and Hervieu Y Y 2014 Diffusion-induced growth of nanowires: generalized boundary conditions and self-consistent kinetic equation J. Cryst. Growth 401 431-40

[28] Vega N C, Wallar R, Caram J, Grinblat G, Tirado M, LaPierre R R and Comedi D 2012 ZnO nanowire co-growth on $\mathrm{SiO} 2$ and $\mathrm{C}$ by carbothermal reduction and vapour advection Nanotechnology 23275602

[29] Matteini F, Tütüncüoglu G, Rüffer D, Alarcon-Llado E and Fontcuberta I Morral F 2014 Ga-assisted growth of GaAs nanowires on silicon, comparison of surface $\mathrm{SiOx}$ of different nature J. Cryst. Growth 404 246-55

[30] Mandl B, Stangl J, Hilner E, Zakharov A A, Hillerich K, Dey A W, Samuelson L, Bauer G, Deppert K and Mikkelsen A 2010 Growth mechanism of self-catalyzed group III-V nanowires Nano Lett. 104443

[31] Priante G, Ambrosini S, Dubrovskii V G, Franciosi A and Rubini A 2013 Stopping and resuming at will the growth of GaAs nanowires Cryst. Growth Des. 133976

[32] Plissard S, Larrieu G, Wallart X and Caroff P 2011 High yield of self-catalyzed GaAs nanowire arrays grown on silicon via gallium droplet positioning Nanotechnology 22275602

[33] Fontcuberta I Morral A, Colombo C, Abstreiter G, Arbiol J and Morante J C 2008 Nucleation mechanism of galliumassisted molecular beam epitaxy growth of gallium arsenide nanowires Appl. Phys. Lett. 92063112

[34] Cirlin G E et al 2010 Self-catalyzed, pure zincblende GaAs nanowires grown on $\mathrm{Si}$ (111) by molecular beam epitaxy Phys. Rev. B 82035302

[35] Venables A, Spiller G D and Hanbucken M 1984 Nucleation and growth of thin films Rep. Prog. Phys. 47399

[36] Bartelt M C and Evans J W 1992 Scaling analysis of diffusionmediated island growth in surface adsorption processes Phys. Rev. B 4612675

[37] Dubrovskii V G and Sibirev N V 2014 Size distributions, scaling properties, and Bartelt-Evans singularities in irreversible growth with size-dependent capture coefficients Phys. Rev. B 89054305

[38] Ramdani M R, Harmand J C, Glas F, Patriarche G and Travers L 2013 Arsenic pathways in self-catalyzed growth of GaAs nanowires Cryst. Growth Design 1391

[39] Dubrovskii V G 2014 Influence of the group V element on the chemical potential and crystal structure of Au-catalyzed IIIV nanowires Appl. Phys. Lett. 104053110

[40] Dubrovskii V G and Nazarenko M V 2010 Nucleation theory beyond the deterministic limit. I. The nucleation stage J. Chem. Phys. 132114507 